\title{
Development of two-dimensional micro-strip gas detector with individual readouts for neutron scattering experiments
}

\section{$\operatorname{AUTHOR}(S):$}

Tanaka, H; Nakamura, T; Yamagishi, H; Soyama, K; Aizawa, K; Ochi, A; Tanimori, T

\section{CITATION:}

Tanaka, H ...[et al]. Development of two-dimensional micro-strip gas detector with individual readouts for neutron scattering experiments. IEEE TRANSACTIONS ON NUCLEAR SCIENCE 2006, 53(4): 2264-2269

\section{ISSUE DATE:}

2006-08

URL:

http://hdl.handle.net/2433/50506

\section{RIGHT:}

(c)2006 IEEE. Personal use of this material is permitted. However, permission to reprint/republish this material for advertising or promotional purposes or for creating new collective works for resale or redistribution to servers or lists, or to reuse any copyrighted component of this work in other works must be obtained from the IEEE. 


\title{
Development of Two-Dimensional Micro-Strip Gas Detector With Individual Readouts for Neutron Scattering Experiments
}

\author{
H. Tanaka, T. Nakamura, H. Yamagishi, K. Soyama, K. Aizawa, A. Ochi, and T. Tanimori
}

\begin{abstract}
We have been developing a Micro-Strip Gas Chamber (MSGC) with individual readouts for neutron scattering experiments. Performance requirements of a two-dimensional position sensitive neutron imaging detector for detecting an accurate Bragg-peak from a sample target include high spatial resolution, high detection efficiency, fast temporal response, and low gamma-ray sensitivity. To meet these specifications in addition to achieving a compact size in spite of the multi-channels in the gas based detector, we constructed a detector system that has a gas vessel with 541 channel feedthrough directly connecting to the data processing circuits. The developed detector system exhibited a pulse pair resolution of less than $1 \mu \mathrm{s}$, a spatial resolution of about $0.8 \mathrm{~mm}$ in full width at half maximum and good agreement between the position of incident neutron and measured peak position.
\end{abstract}

Index Terms-High spatial resolution, individual readouts, micro-strip, position sensitive neutron detector.

\section{INTRODUCTION}

$\mathbf{N}$ EUTRON imaging detectors are being extensively developed for neutron scattering experiments [1] using high-intensity pulsed neutron sources(under construction in Japan [2], the United States [3], and the United Kingdom [4]). Characteristic neutron detector requirements for such spectrometers as a reflectometer, in small angle neutron scattering experiments include superior spatial resolution $<1 \mathrm{~mm}$, detection efficiency $>80 \%$ (for $1.8 \AA$ ), high count rate capability $>10^{6} \mathrm{cps} /$ detector as global and $>10^{5} \mathrm{cps} / \mathrm{mm}^{2}$ as local, neutron-gamma-ray discrimination ratio $<10^{-8}$, and sensitive area $>100 \mathrm{~cm}^{2}$.

To satisfy these detector specifications, we have been developing a two-dimensional position sensitive neutron gas detector system with individual readouts [5], [6]. In addition to these specifications, a Micro-Strip Gas Chamber (MSGC) [7] that exhibits better reproducibility of fabrication and mechanical stability of the readout strips than a multi-wire detection head was used. The developed detector system has a gas vessel with multi-channel feedthrough measured $\phi 350 \times 80 \mathrm{~mm}$ that seals any gas mixture up to 11-atm, a electric circuit board for multi-

Manuscript received January 25, 2006; revised April 17, 2006.

H. Tanaka is with the Research Reactor Institute, Kyoto University, Osaka 590-0494, Japan (e-mail: h-tanaka@ rri.kyoto-u.ac.jp).

T. Nakamura, H. Yamagishi, K. Soyama, and K. Aizawa are with the Quantum Beam Science Directorate, Japan Atomic Energy Agency, Tokai, Naka, Ibaraki 319-1195, Japan.

A. Ochi is with the Department of Physics, Graduate School of Science and Technology, Kobe University, Kobe 657-8501, Japan.

T. Tanimori is with the Cosmic-Ray Group, Department of Physics, Graduate School of Science, Kyoto University, Kyoto 606-8502, Japan.

Digital Object Identifier 10.1109/TNS.2006.875841

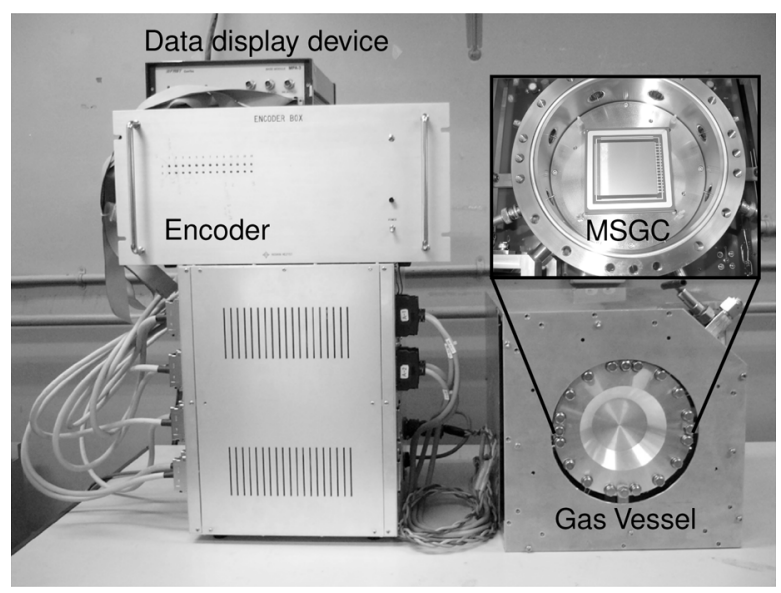

Fig. 1. Photograph of the individual readout system made up of a gas vessel, a Micro-Strip Gas Chamber (MSGC), an encoder and a data display device.

channel data processing with fast temporal response and an encoder system for detecting the position of incident neutrons.

In this report we detail the performance of the individual readout system with MSGC we have developed; finding such characteristics as a pulse pair resolution $<1 \mu \mathrm{s}$, spatial resolution $\sim 0.8 \mathrm{~mm}$, good agreement between the incident and measured positions, and counting uniformity $<7 \%$. Furthermore, the detection efficiency for thermal neutrons was estimated to be greater than $80 \%$ from calculations considering the helium gas and the conversion gap of $22 \mathrm{~mm}$.

\section{INDIVIDUAL READOUT SYSTEM}

Fig. 1 is a photograph of our individual readout system that comprises an MSGC of $51 \times 51 \mathrm{~mm}^{2}$ developed for X-ray imaging experiments [8], a gas vessel that can withstand up to 11-atm and which has 541 channel signal feedthrough and eight high voltage connectors, an encoder system for calculating the position of incident neutrons, and a data acquisition and display device.

Fig. 2 shows a schematic layout of the data processing system with individual readouts. The ASD board implementing 16 ASD-ASICs [9] has 64 channel signal inputs and two 32 channel Low-Voltage-Differential-Signaling (LVDS) outputs. Secondary particles generated by the nuclear reaction of ${ }^{3} \mathrm{He}(\mathrm{n}, \mathrm{p}) \mathrm{t}$ were isotropically emitted and ionized the gas medium in a conversion gap of $22 \mathrm{~mm}$ width. The electrons ionized along the track of the secondary particles drift toward the anode strips because of the electronic field of $2.5 \mathrm{kV} / \mathrm{cm}$. 


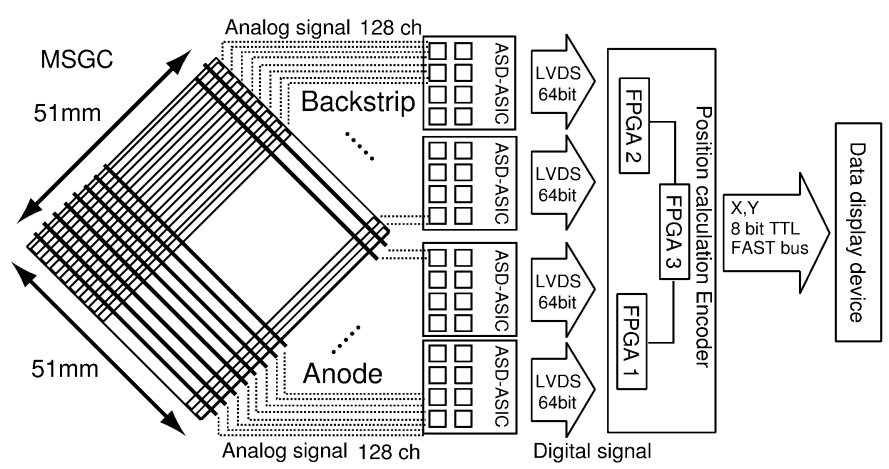

Fig. 2. Schematic layout of an individual signal circuit. The system we developed has an analog [processed by an Amplifier-Shaper-Discriminator (ASD)ASIC) and a digital (processed by an FPGA) circuit.
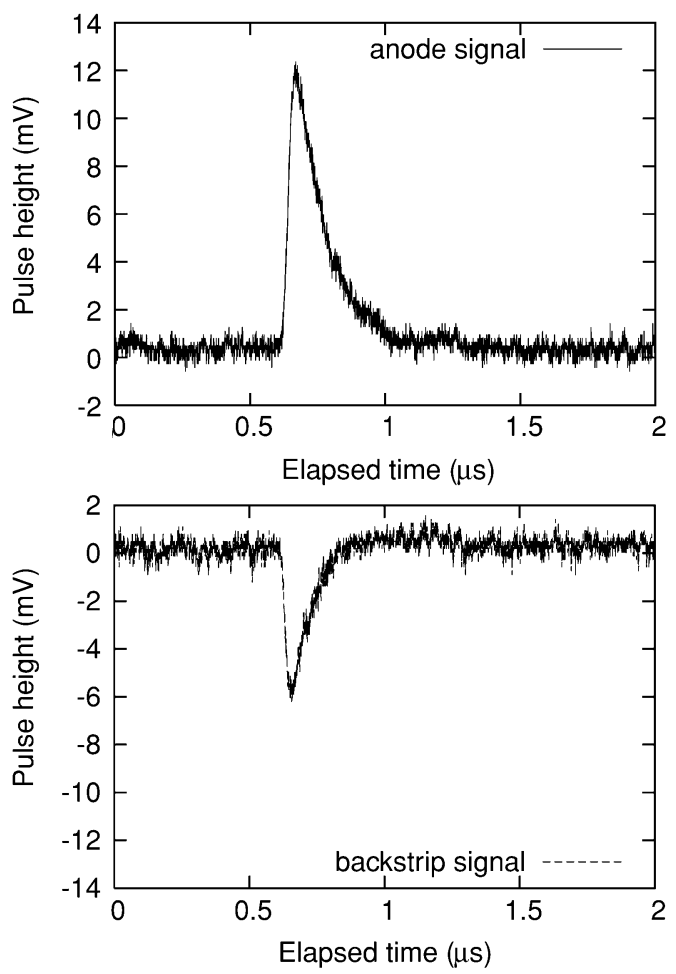

Fig. 3. Typical pulse shapes produced by neutron irradiation at the anode and backstrip amplified and shaped by ASD-ASIC.

Gas multiplication occurs near the thin anode strips of $10 \mu \mathrm{m}$ formed on the polyimide film of $17 \mu \mathrm{m}$ thickness. Simultaneously, signals are induced in backstrips positioned orthogonal to the anode strips and at the rear of the polyimide film. Each pulsed current signal is individually amplified, shaped with an integral time constant of $80 \mathrm{~ns}$, and discriminated by ASD-ASICs. Typical ASD analog pulse shapes for anodes and backstrips, before discrimination, are shown in Fig. 3. A pulse width of $\sim 200 \mathrm{~ns}$ and a good signal to noise ratio were confirmed. A pulse pair resolution of this system was less than $1 \mu \mathrm{s}$, which include the data processing and encoding time of $600 \mathrm{~ns}$ at present settings.

Fig. 4 shows the calculation of the energy deposit of a proton $(574 \mathrm{keV})$ and a triton $(191 \mathrm{keV})$ that proceed orthogonal to the anode direction in a gas mixture of 6.4-atm helium with 1.6-atm $\mathrm{CF}_{4}$. We assume that each value multiplied by a certain

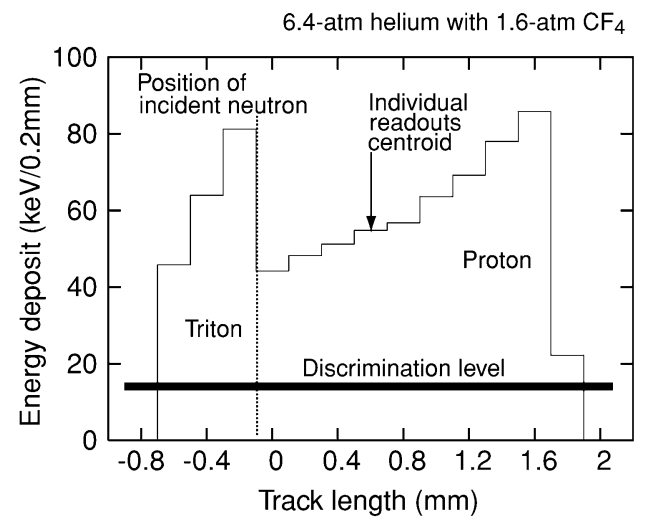

Fig. 4. Calculated energy deposit of a proton $(574 \mathrm{keV})$ and a triton $(191 \mathrm{keV})$ in a gas mixture of 6.4-atm helium with 1.6-atm $\mathrm{CF}_{4}$. The spatial resolution is derived from the displacement from the position of the incident neutron to the centroid of individual readouts.

gas gain constant can be taken to be the value of the signal induced on each of the anode strips. To ensure a sufficient signal to noise ratio, we connect two neighboring strips of $0.2 \mathrm{~mm}$ pitch with wire bonding corresponding to a $0.4 \mathrm{~mm}$ readout pitch. According to this calculation, the ranges for protons and tritons are roughly estimated to be $1.8 \mathrm{~mm}$ and $0.6 \mathrm{~mm}$, respectively. The displacement between the position of incident neutrons and the centroid of the total range is calculated to $0.6 \mathrm{~mm}$. Because protons and tritons are isotropically emitted, the position resolution is expected to less than $1.2 \mathrm{~mm}$, considering the symmetry with respect to the position of incident neutrons.

Any channel over the discrimination level is treated as a "hit channel". The LVDS output of the hit channel bit array is fed into the encoder system, which calculates the edge of the secondary particle tracks. The encoder is comprised of three FieldProgrammable-Gate-Arrays (FPGA) operating at $20 \mathrm{MHz}$. Two (FPGA1 and FPGA2) of the three FPGAs process the anodeand backstrip-direction signals, while the other FPGA (FPGA3) has an AND circuit for the anode and backstrip to confirm the neutron event. Since each FPGA can handle 256 signals, this system easily expands to $256 \times 256$ channel readouts. Both of the edge channels in the anode and backstrip direction are sent to a data display and store device (MPA-3 system, FAST Comtec Co.). This encoder can discriminate neutrons and gamma-rays by rejecting the events having a larger number of hit channels than a variable preset number of hit channels. The rejection of gamma-ray events is based on the difference of the track length corresponding to the number of hit channels of Compton electrons (for gamma-ray) and protons and tritons (for neutrons) expected for the total range.

Further, this system can carry out Time of Flight (ToF) measurements using timing signals from the neutron chopper system or accelerator.

\section{EXPERIMENTAL RESULTS}

\section{A. Fundamental Measurements}

To evaluate the developed individual readout system, gas gain and plateau measurements were performed. Fig. 5 shows the pulse height distribution of the sum of the cathode strip signals. 


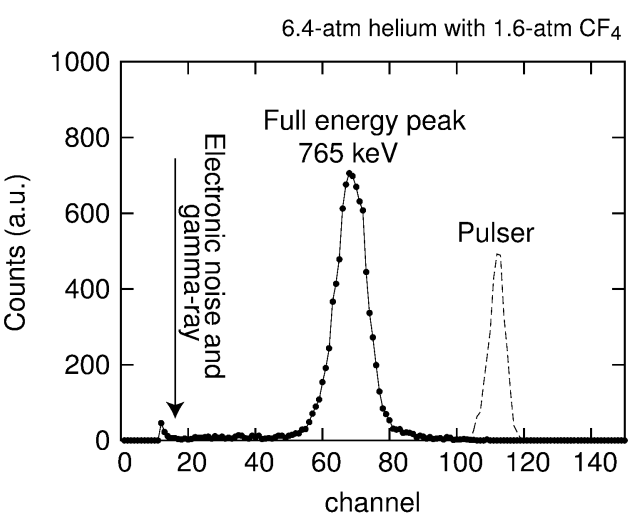

Fig. 5. Pulse height distribution of the sum of cathode strip signals. The dash line shows the response of the pulser.

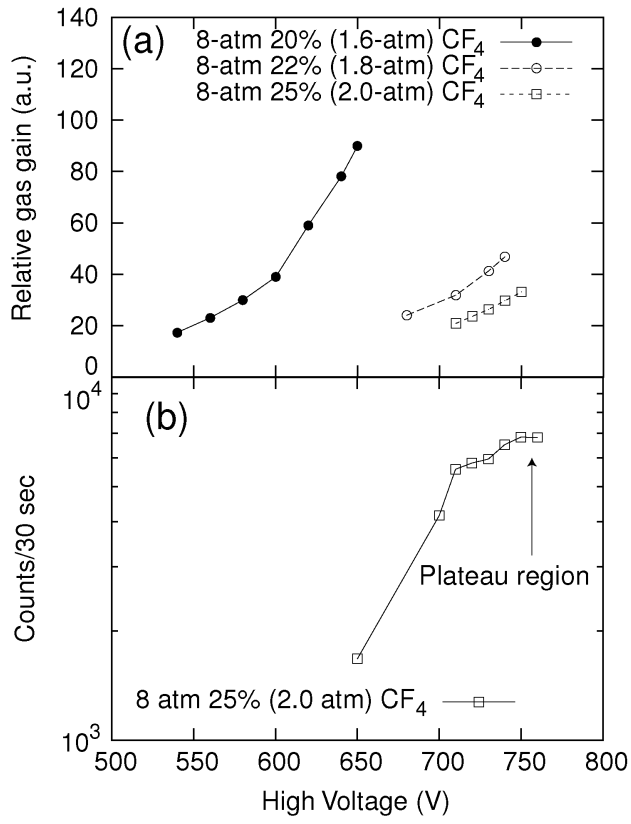

Fig. 6. (a) The relative gas gain as a function of the high voltage applied to the anode. (b) The plateau measurements under a gas mixture of 8 -atm $25 \%$ (2.0-atm) $\mathrm{CF}_{4}$. These measurements were carried out by varying the amount of $\mathrm{CF}_{4}$ as the stopping gas. Helium gas was filled so as to maintain the total gas pressure at 8 -atm.

The energy resolution of a full energy peak of $765 \mathrm{keV}$ is $15 \%$, which is consistent with the values of micro pattern neutron detectors measured elsewhere [10]. The full energy peak is clearly separated from the electronic noise and gamma-ray events. This system can operate with a low background by the removal of the events having a larger number of hit channels and executing pulse height distribution discrimination (shown in Fig. 5).

Fig. 6 shows (a)the gas gain under three gas mixtures and (b)the plateau measurements under the greatest percentage of the stopping gas mixture of 8 -atm $25 \%\left(2.0\right.$-atm) $\mathrm{CF}_{4}$ in these three gas mixtures as a function of high voltage applied to the anode. In Fig. 6 the plateau is exhibited at the applied voltage of $750 \mathrm{~V}$ and a relative gas gain of 30 . It can be expected that plateaus are also exhibited under other gas mixtures with a relative gas gain larger than 30 .

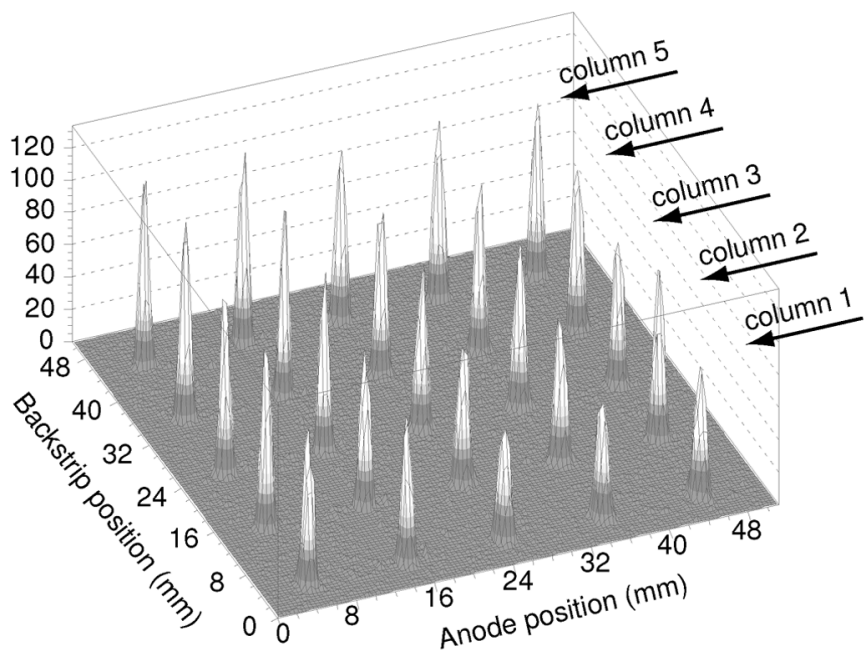

Fig. 7. Counting uniformity measurements carried out using a collimated neutron beam of $0.3 \times 0.3 \mathrm{~mm}^{2}$.

The detection efficiency for thermal neutrons was estimated to be larger than $84 \%$ under each gas mixture, from calculations that considered the helium gas and the conversion gap of $22 \mathrm{~mm}$.

\section{B. Linearity and Counting Uniformity Results}

For the neutron scattering experiment, accurate information about the Bragg-peak position and the peak intensity are required. To evaluate the detector performance such as position linearity and counting uniformity, the well collimated cold neutron beam of the JRR-3 reactor in Japan Atomic Energy Agency was used. These experiments were performed under a gas mixture of 6.4-atm helium with 1.6-atm $\mathrm{CF}_{4}$ and with an applied high voltage of $650 \mathrm{~V}$ (relative gas gain of 90). Fig. 7 shows the results of a raster scan using a $0.3 \times 0.3 \mathrm{~mm}^{2}$ pencil neutron beam. The deviation including a statistical error of $3 \%$ from the mean, indicated that counting uniformity was less than $\pm 7 \%$ without correction.

Fig. 8 shows the linearity between the measured and the actual incident positions. To ensure accurate measurements, a collimated beam of $0.2 \times 10 \mathrm{~mm}^{2}$ was used. The incident position was changed by the pulse motor with a step by step raster scan of $1 \mathrm{~mm}$ pitch, and the measured peak position was derived from gaussian fitting along both (a)anode (irradiated at the 16-26 $\mathrm{mm}$ position of the backstrip) and (b)backstrip (irradiated at the 17-27 $\mathrm{mm}$ position of the anode) directions.

The experimental results were fitted by a linear function, and respective slopes of the anode- and backstrip- were $1.0016 \pm$ 0.0004 and $1.0106 \pm 0.0009$. Fig. 9 shows that the deviation of the measured position from the linear fitting in the anode- and backstrip- directions was $\pm 0.5 \%$. The displacement in the backstrip-direction is larger than that in the anode-direction, because the backstrip has a width of $0.1 \mathrm{~mm}$. The Sub-Section 3 below also indicates that the spatial resolution in the backstrip direction is worse than that in the anode direction.

\section{Gamma Sensitivity}

Gamma-ray sensitivity measurements were carried out using a ${ }^{60} \mathrm{Co}$ gamma-ray source of $3.7 \times 10^{6} \mathrm{~Bq}$ located near the de- 


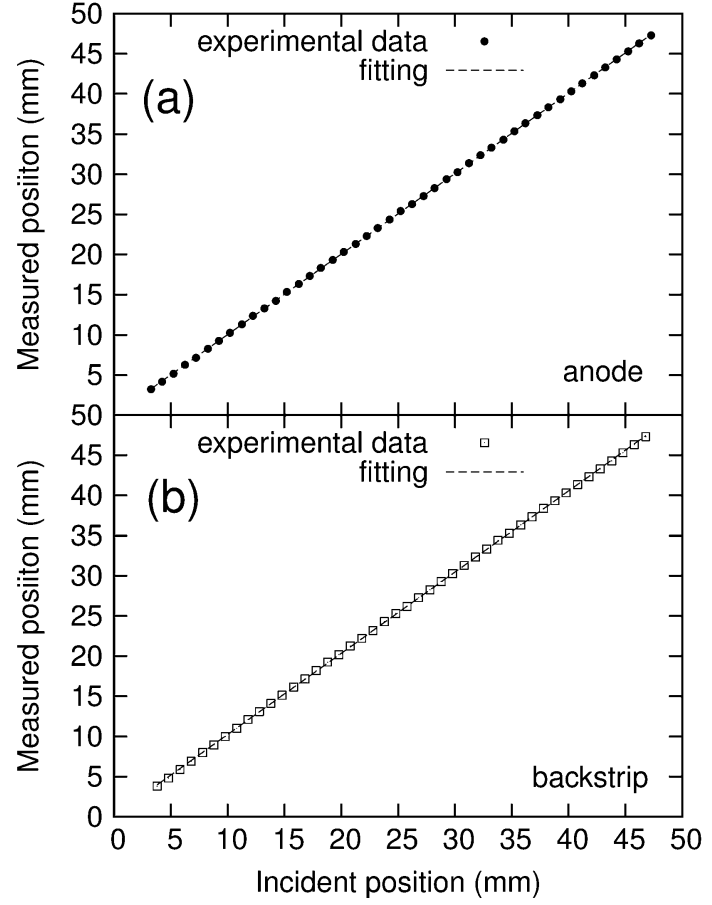

Fig. 8. The measured position as a function of the actual position of an incident neutron. This measurements were performed by a rectangular beam of $0.2 \times 10 \mathrm{~mm}^{2}$.

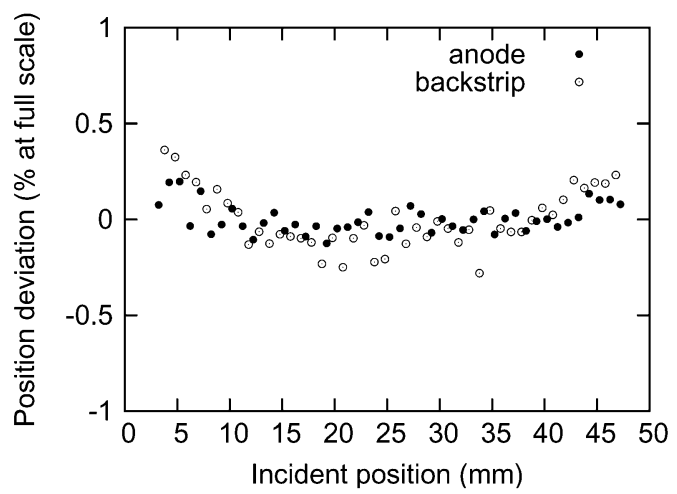

Fig. 9. The position deviation from a linear fitting as a function of the incident position over the full scale of $50 \mathrm{~mm}$.

tector window. The relative gas gain of 90 described above and the discrimination level were set. The ${ }^{60} \mathrm{Co}$ gamma source emits two gamma-rays (1.1 and $1.3 \mathrm{MeV}$ ) per one disintegration; the photon flux reaching the sensitive area of $25 \mathrm{~cm}^{2}$ was roughly estimated as $8.5 \times 10^{5}$ (photons/s), considering the detection area and the solid angle defined by the distance between the sensitive area and the gamma-ray source. The measured counting rate was $0.13 \mathrm{cps}$; therefore, gamma-ray sensitivity was estimated to be $6.1 \times 10^{-9}$ (counts/photon/ $/ \mathrm{cm}^{2}$ ).

\section{Spatial Resolution}

To evaluate the spatial resolution, the uniformity experiment data shown in Fig. 7 were used. Fig. 10 shows the spatial resolution in the (a)anode and (b)backstrip directions of each of the

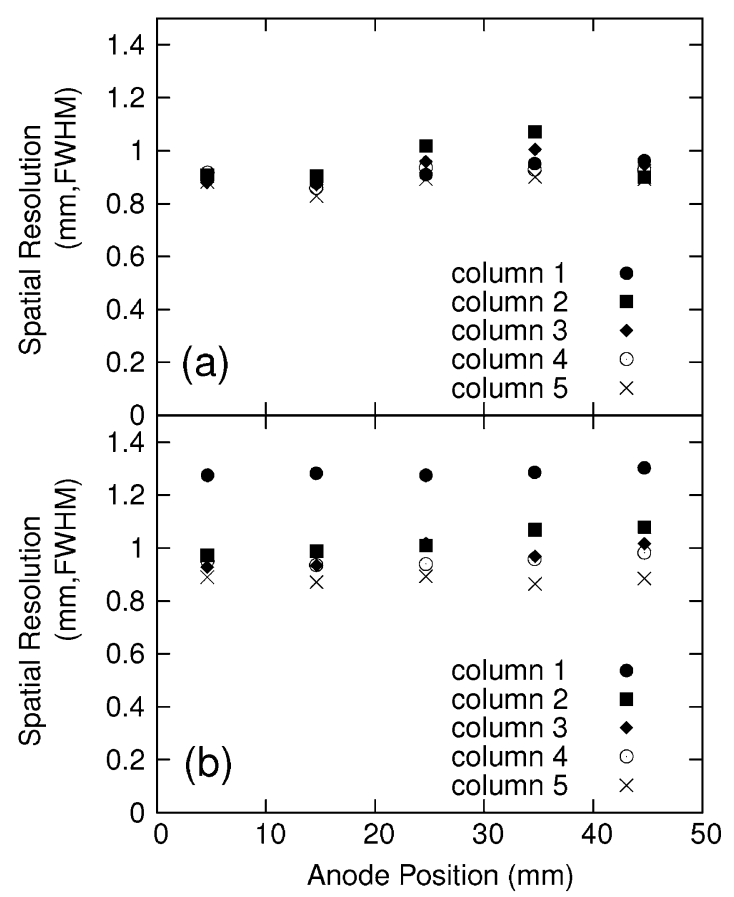

Fig. 10. Spatial resolution over the whole area in the (a) anode and (b) backstrip directions. The number of each column corresponds to the incident position shown in Fig. 7.

positions of incident neutrons. Columns 1, 2, 3, 4, and 5 correspond to the backstrip positions of $5,15,25,35$, and $45 \mathrm{~mm}$, respectively, from the edge of the detection head as shown in Fig. 7.

The spatial resolutions in the anode and backstrip directions were roughly uniform $(\sim 1 \mathrm{~mm})$ except for in column 1 in the backstrip direction. This shift was attributed to the fact that there was distortion of the electric field caused by the bonding pad of the cathode positioned near column 1 .

Because of the reduction of the total range (especially proton range) caused by the wall effect at the drift plate and the MSGC surface, the experimental results are lower than the expected spatial resolution of $1.2 \mathrm{~mm}$ described in the Section II. This phenomenon could be due to the $0.4 \mathrm{~mm}$ pitch of the anode or backstrip.

High spatial resolution is necessary to discriminate each Bragg peak. To attempt a measurement with higher spatial resolution, a narrow neutron beam of $0.2 \times 10 \mathrm{~mm}^{2}$ was used. Measurements were carried out under a gas mixture of 6-atm helium with 2-atm $\mathrm{CF}_{4}$ with a relative gas gain of 30, as shown in Fig. 6. Fig. 11 shows a neutron image resulting from irradiation over the whole area to evaluate the spatial resolution in the anode direction. To maintain the same beam conditions, the detector was rotated 90 degrees to measure spatial resolution in the backstrip direction.

Figs. 12 and 13 show spatial resolution in the anode and backstrip directions at each of the positions of incident neutrons. The top in Fig. 12 shows the spatial resolution in the anode direction at each incident position shown in Fig. 11. The bottom figure shows the typical anode projection generated by irradiation the 20-30 mm region of the backstrip. Similarly, the top figure in 


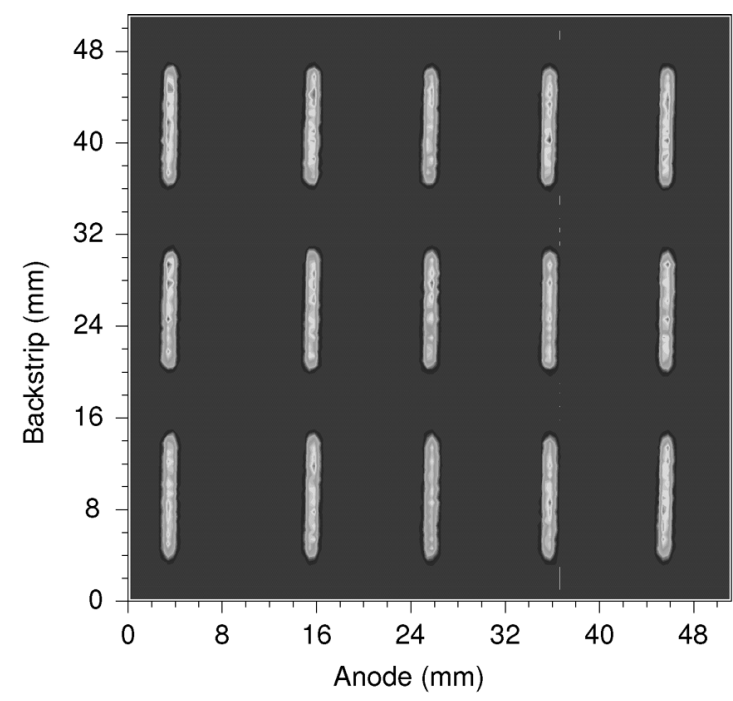

Fig. 11. Neutron imaging using a rectangular beam of $0.2 \times 10 \mathrm{~mm}^{2}$ to evaluate spatial resolution in the anode direction.

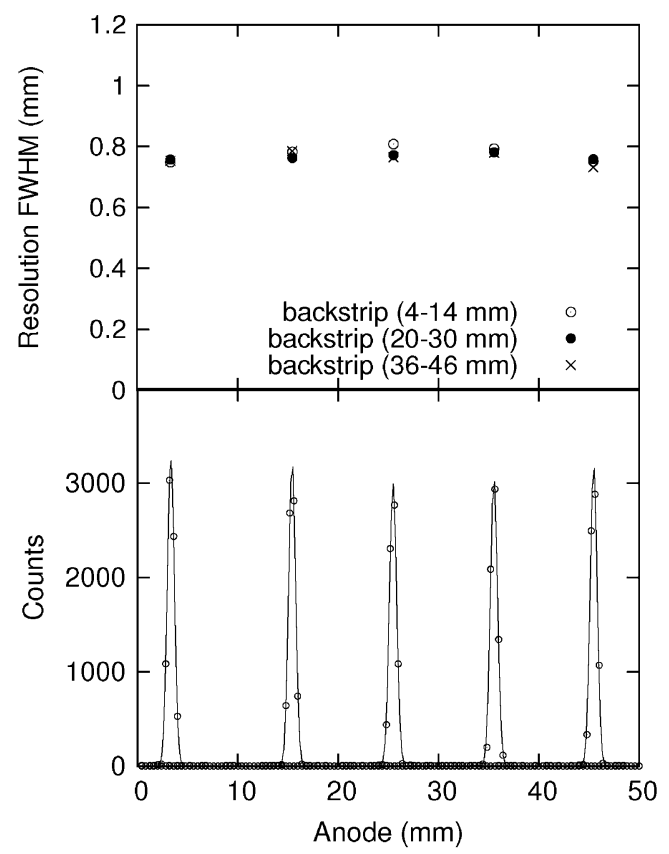

Fig. 12. Spatial resolution over the whole area in the anode direction (top). Anode projection generated by irradiation over the $20-30 \mathrm{~mm}$ region of the backstrip (bottom) shown by filled circles in the top figure.

Fig. 13 shows spatial resolution in the backstrip direction, while the bottom figure shows a typical backstrip projection generated in the 4-14 $\mathrm{mm}$ region of the anode.

The spatial resolutions in the anode and backstrip directions are roughly uniform, no more than $0.8 \mathrm{~mm}$, except at the edge in the backstrip direction.

In future work, we plan to carry out quantitative measurements of gamma-ray sensitivity. Further, we have learned of a new detection method to decide the position of incident neutrons; this uses the difference of secondary particle tracks among the individual readouts [11], [12], and a demonstration of a test of this method will be performed.

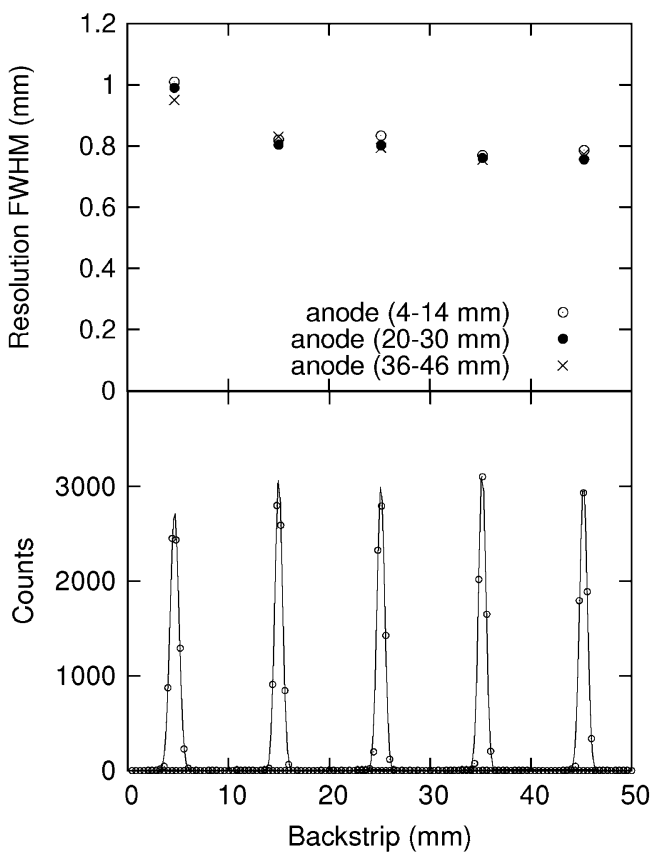

Fig. 13. Spatial resolution over the whole area in the backstrip direction (top). Backstrip projection generated by irradiation over the 4-14 $\mathrm{mm}$ region of the anode (bottom) shown by open circles in the top figure.

\section{CONCLUSION}

We constructed a two-dimensional micro-strip gas neutron detector with individual readouts for neutron scattering experiments. The performance of this developed system was confirmed with a collimated neutron beam. According to the results of the plateau and gas gain measurements, the detection efficiency is expected to be larger than $80 \%$ for thermal neutrons. Spatial resolution in both anode and backstrip directions was about $0.8 \mathrm{~mm}$, except at the edge of the sensitive area, with a detection uniformity of $\pm 7 \%$. Further, superior position linearity of $\pm 0.5 \%$ was found in both anode and backstrip directions.

\section{ACKNOWLEDGMENT}

The authors would like to thank for Dr. Yamazaki, Dr. Moriai, and Dr. Tamura of JAEA for assisting our experiments at the JRR-3 reactor.

\section{REFERENCES}

[1] J. C. Buffet, "Advances in detectors for single crystal neutron diffraction," Nucl. Instrum. Methods Phys. Res. A, vol. A554, pp. 392-405, 2005.

[2] [Online]. Available: http://j-parc.jp

[3] [Online]. Available: http://www.sns.gov

[4] [Online]. Available: http://www.isis.rl.ac.uk

[5] H. Tanaka, T. Nakamura, H. Yamagishi, K. Soyama, and K. Aizawa, "A two-dimensional gas detector with individual readouts for neutron detection with a high spatial resolution and fast temporal response," Rev. Sci. Instrum., vol. 76, p. 093302, 2005.

[6] T. Nakamura, H. Tanaka, H. Yamagishi, K. Soyama, and K. Aizawa, Nucl. Instrum. Methods Phys. Res. A, submitted for publication.

[7] A. Oed, "Position-sensitive detector with microstrip anode for electron multiplication with gases," Nucl. Instrum. Methods Phys. Res. A, vol. A263, pp. 351-359, 1988. 
[8] T. Tanimori, A. Ochi, S. Minami, and T. Nagae, "Development of an imaging microstrip gas chamber with a $5 \mathrm{~cm} \times 5 \mathrm{~cm}$ area based on multi-chip module technology," Nucl. Instrum. Methods Phys. Res. A, vol. A381, pp. 280-288, 1996.

[9] R. Orito, "Development of an ASD IC for the micro pixel chamber," IEEE Trans. Nucl. Sci., vol. 51, no. 4, pp. 1337-1342, Aug. 2004.

[10] J. F. Clergeau, "Operation of sealed microstrip gas chambers at the ILL," Nucl. Instrum. Methods Phys. Res. A, vol. A471, pp. 60-68, 2001.
[11] H. Yamagishi, T. Nakamura, K. Soyama, S. Masaoka, and K. Aizawa, "Novel instrument system for discriminating secondary particles in high-spatial-resolution neutron detection," Rev. Sci. Instrum., vol. 75, pp. 2340-2345, 2004.

[12] T. Nakamura, H. Yamagishi, S. Masaoka, K. Soyama, and K. Aizawa, "Development of a high-performance microstrip gas chamber with a capability of track discrimination for neutron detection," Nucl. Instrum. Methods Phys. Res. A, vol. A529, pp. 336-341, 2004. 